\title{
Positive asymptotically almost periodic solutions of an impulsive hematopoiesis model
}

\author{
Peng Chen ${ }^{1}$, Hui-Sheng Ding ${ }^{1}$, Gaston M. N'Guérékata ${ }^{2}$ \\ 1 College of Mathematics and Information Science, \\ Jiangxi Normal University, \\ Nanchang, Jiangxi 330022, People's Republic of China. \\ ${ }^{2}$ Department of Mathematics, \\ Morgan State University, \\ 1700 E. Cold Spring Lane, Baltimore, M.D. 21251, USA.
}

9972740999@qq.com, dinghs@mail.ustc.edu.cn, Gaston.N'Guerekata@morgan.edu

\begin{abstract}
In this paper, we introduce the notion of impulsive asymptotically almost periodic functions and prove some basic properties of such functions. Then, we discuss the existence and exponential stability of positive asymptotically almost periodic solution for an impulsive hematopoiesis model. An example is given to illustrate our results.
\end{abstract}

\section{RESUMEN}

En este artículo, introducimos la noción de funciones impulsivas asintóticamente casi periódicas y probamos algunas propiedades básicas para dichas funciones. Luego, discutimos la existencia y estabilidad exponencial de soluciones positivas asintóticamente casi periódicas para un modelo impulsivo de hematopoyesis. Un ejemplo es dado para ilustrar nuestros resultados.

Keywords and Phrases: Almost periodic, asymptotically almost periodic, impulsive, hematopoiesis. 2010 AMS Mathematics Subject Classification: 34K14. 


\section{$1 \quad$ Introduction and preliminaries}

In [8], Mackey and Glass proposed the following nonlinear delay differential equation

$$
h^{\prime}(t)=-\alpha h(t)+\frac{\beta}{1+h^{\mathfrak{n}}(t-\tau)}
$$

as an appropriate model of hematopoiesis that describes the process of production of all types of blood cells generated by a remarkable self-regulated system that is responsive to the demands put upon it. In medical terms, $h(t)$ denotes the density of mature cells in blood circulation at time $t$ and $\tau$ is the time delay between the production of immature cells in the bone marrow and their maturation for release in circulating bloodstream. It is assumed that the cells are lost from the circulation at a rate $\alpha$, and the flux of the cells into the circulation from the stem cell compartment depends on the density of mature cells at the previous time $t-\tau$.

In this paper, we consider the existence and stability of asymptotically almost periodic solutions for the following impulsive hematopoiesis model:

$$
\begin{cases}x^{\prime}(t)=-a(t) x(t)+\frac{b(t)}{1+x^{n}(t-\tau)}, & t \neq t_{k}, \\ \left.\Delta x\right|_{t=t_{k}}=c_{k} x\left(t_{k}\right)+I_{k}\left(x\left(t_{k}\right)\right), & k \in \mathbb{Z}\end{cases}
$$

where $\left.\Delta x\right|_{t=t_{k}}=x\left(t_{k}+0\right)-x\left(t_{k}-0\right), \tau \geq 0$ is a constant and the coefficients satisfy some conditions, which will be listed in Section 3 .

The direct impetus of this paper comes from two sources. The first source is some recent works on the almost periodic solutions for hematopoiesis models without impulse effect (see, e.g., $[4,7]$ and references therein); the second source is some recent works on periodic solutions and almost periodic solutions for impulsive hematopoiesis models (see, e.g., $[10,1,12]$ and references therein). Stimulated by these works, we aim to make further study on this topic. As one will see, there are two differences of our work from some earlier works on almost periodic solutions to equation (1.2) (cf. [1,12]). The first difference is that we do not assume that $\inf _{t \in \mathbb{R}} a(t)>0$ and $-1 \leq c_{k} \leq 0$ for all

$k \in \mathbb{Z}$. In fact, we weaken these assumptions to $M(a):=\lim _{T \rightarrow+\infty} \frac{1}{T} \int_{0}^{T} a(t) d t>0$ and $-1 \leq c_{k}$ for all $k \in \mathbb{Z}$. The second difference is that we investigate the existence and stability of asymptotically almost periodic solution to equation (1.2). To the best of our knowledge, it seems that until now there is no results concerning asymptotically almost periodic solution to equation (1.2). Recall that in 1940s, M. Fréchet [5] introduced the notion of asymptotically almost periodicity, which turns out to be one of the most interesting and important generalizations of almost periodicity.

Throughout this paper, we denote by $\mathbb{R}$ the set of real numbers, by $\mathbb{R}^{+}$the set of nonnegative real numbers, by $\mathbb{Z}$ the set of integers, and by $\mathbb{N}$ the set of positive integers.

Now, let us recall some basic notations about classical almost periodic type functions (for more details, we refer the reader to $[2,3])$. 
Definition 1.1. A set $\mathrm{P} \subset \mathbb{Z}$ (or $\mathbb{R}$ ) is called relatively dense in $\mathbb{Z}$ (or $\mathbb{R}$ ) if there exists a number $\mathrm{l} \in \mathbb{N}\left(\right.$ or $\mathbb{R}^{+}$) such that $\forall \mathrm{a} \in \mathbb{Z}$ (or $\left.\mathbb{R}\right),[\mathrm{a}, \mathrm{a}+\mathrm{l}] \cap \mathrm{P} \neq \varnothing$.

Definition 1.2. A continuous function $f: \mathbb{R} \rightarrow \mathbb{R}$ is called almost periodic if for every $\varepsilon>0$,

$$
P(\varepsilon, f)=\left\{\tau \in \mathbb{R}: \sup _{t \in \mathbb{R}}|f(t+\tau)-f(t)|<\varepsilon\right\}
$$

is relatively dense in $\mathbb{R}$. We denote the set of all such functions by $\mathrm{AP}(\mathbb{R})$.

Definition 1.3. A sequence $\mathrm{f}: \mathbb{Z} \rightarrow \mathbb{R}$ is called almost periodic if for every $\varepsilon>0$,

$$
P(\varepsilon, f)=\left\{\tau \in \mathbb{Z}: \sup _{n \in \mathbb{Z}}|f(n+\tau)-f(n)|<\varepsilon\right\}
$$

is relatively dense in $\mathbb{Z}$. We denote the set of all such sequences by $\mathrm{AP}(\mathbb{Z})$.

Definition 1.4. A sequence $\mathrm{f}: \mathbb{Z} \rightarrow \mathbb{R}$ is called asymptotically almost periodic if $\mathrm{f}=\mathrm{g}+\mathrm{h}$, where $\mathrm{g} \in \mathrm{AP}(\mathbb{Z})$ and $\mathrm{h} \in \mathrm{C}_{0}(\mathbb{Z})$, where $\mathrm{C}_{0}(\mathbb{Z})$ is the set of all functions $\mathrm{h}: \mathbb{Z} \rightarrow \mathbb{R}$ with $\lim _{\mathrm{n} \rightarrow \infty} \mathrm{h}(\mathrm{n})=0$. We denote the set of all such sequences by $\mathrm{AAP}(\mathbb{Z})$.

Definition 1.5. A set of sequences $\mathrm{f}_{\lambda}: \mathbb{Z} \rightarrow \mathbb{R}, \lambda$ belongs to some index set $\Lambda$, is called equi-almost periodic if for every $\varepsilon>0, \bigcap_{\lambda \in \Lambda} \mathrm{P}\left(\varepsilon, \mathrm{f}_{\lambda}\right)$ is relatively dense in $\mathbb{Z}$.

Next, let us recall some basic notations and properties about impulsive almost periodic type functions (for more details, we refer the reader to $[9,11,6]$ ). 0 , and

Let $\mathcal{T}$ be the set of all sequences $\left\{t_{k}\right\}_{k \in \mathbb{Z}} \subset \mathbb{R}$ satisfying $t_{k}<t_{k+1}$ for all $k \in \mathbb{Z}, \inf _{k \in \mathbb{Z}}\left|t_{k+1}-t_{k}\right|>$

$$
\lim _{k \rightarrow+\infty} t_{k}=+\infty, \quad \lim _{k \rightarrow-\infty} t_{k}=-\infty .
$$

It is easy to see that for every $T=\left\{t_{k}\right\}_{k \in \mathbb{Z}} \in \mathcal{T}$ and $a \in \mathbb{R}$, there holds $T+a \in \mathcal{T}$. In addition, we denote by $\mathcal{P}_{\mathrm{T}} \mathrm{C}(\mathbb{R})$ the set of all functions $f: \mathbb{R} \rightarrow \mathbb{R}$ such that $f$ is continuous on $\mathbb{R} \backslash T$, and for every $t_{k} \in T, f\left(t_{k}-0\right)=f\left(t_{k}\right)$ and $f\left(t_{k}+0\right)$ exists.

Definition 1.6. Let $\mathrm{T}=\left\{\mathrm{t}_{\mathrm{k}}\right\}_{\mathrm{k} \in \mathbb{Z}} \in \mathcal{T}$. A function $\mathrm{f} \in \mathcal{P}_{\mathrm{T}} \mathrm{C}(\mathbb{R})$ is said to be almost periodic if

(i) the set of sequences $\left\{\mathrm{T}_{j}\right\}_{j \in \mathbb{Z}}$ is equi-almost periodic, where $\mathrm{T}_{\mathrm{j}}=\left\{\mathrm{t}_{\mathrm{k}}^{\mathrm{j}}: \mathrm{t}_{\mathrm{k}}^{\mathrm{j}}=\mathrm{t}_{\mathrm{k}+\mathrm{j}}-\mathrm{t}_{\mathrm{k}}, \mathrm{k} \in \mathbb{Z}\right\}$ for every $\mathbf{j} \in \mathbb{Z}$;

(ii) for every $\varepsilon>0$, there exists $\delta>0$ such that if $\mathrm{t}^{\prime}$ and $\mathrm{t}^{\prime \prime}$ belong to the same interval of continuity of $\mathrm{f}$ and $\left|\mathrm{t}^{\prime}-\mathrm{t}^{\prime \prime}\right|<\delta$, then $\left|\mathrm{f}\left(\mathrm{t}^{\prime}\right)-\mathrm{f}\left(\mathrm{t}^{\prime \prime}\right)\right|<\varepsilon$;

(iii) for every $\varepsilon>0$, there exists a relatively dense set $\mathrm{P}(\varepsilon, \mathbf{f})$ in $\mathbb{R}$ such that $|\mathrm{f}(\mathrm{t}+\mathrm{r})-\mathbf{f}(\mathrm{t})|<\varepsilon$ for every $\mathrm{r} \in \mathrm{P}(\varepsilon, \mathrm{f})$ and every $\mathrm{t} \in \mathbb{R}$ with $\left|\mathrm{t}-\mathrm{t}_{\mathrm{k}}\right|>\varepsilon$ for all $\mathrm{k} \in \mathbb{Z}$.

We denote the set of all such functions by $\mathcal{P}_{\mathrm{T}} \mathrm{AP}(\mathbb{R})$. 
Lemma 1.7. Let $\mathrm{T}=\left\{\mathrm{t}_{\mathrm{k}}\right\}_{\mathrm{k} \in \mathbb{Z}} \in \mathcal{T}$, and $\mathrm{f}, \mathrm{g} \in \mathcal{P}_{\mathrm{T}} \mathrm{AP}(\mathbb{R})$. Then, the following assertions hold true:

(i) $\mathrm{f}+\mathrm{g} \in \mathcal{P}_{\mathrm{T}} \mathrm{AP}(\mathbb{R})$ and $\mathrm{f} \cdot \mathrm{g} \in \mathcal{P}_{\mathrm{T}} \mathrm{AP}(\mathbb{R})$.

(ii) $\mathrm{f} / \mathrm{g} \in \mathcal{P}_{\mathrm{T}} \mathrm{AP}(\mathbb{R})$ provided that $\inf _{\mathbf{t} \in \mathbb{R}}|\mathrm{g}(\mathrm{t})|>0$.

(iii) $\mathrm{k} \rightarrow \mathrm{f}\left(\mathrm{t}_{\mathrm{k}}\right)$ belongs to $\mathrm{AP}(\mathbb{Z})$.

(iv) $\mathcal{P}_{\mathrm{T}} \mathrm{AP}(\mathbb{R})$ is a Banach space under the supremum norm.

(v) for every $\mathrm{a} \in \mathbb{R}, \mathrm{f}(\cdot-\mathrm{a}) \in \mathcal{P}_{\mathrm{T}+\mathrm{a}} \mathrm{AP}(\mathbb{R})$.

Proof. (i)-(iii) have been proved in [9]. Moreover, by definition of $\mathcal{P}_{\mathrm{T}} \mathrm{AP}(\mathbb{R})$, it is not difficult to prove (iv) and (v). Here, we omit the details.

Remark 1.8. It is not difficult to show that a continuous function $f \in \mathcal{P}_{\mathrm{T}} A P(\mathbb{R})$ implies that $f \in A P(\mathbb{R})$.

We denote the set of all functions $f \in \mathcal{P}_{\mathrm{T}} \mathrm{C}(\mathbb{R})$ with $\lim _{\mathrm{t} \rightarrow \infty} \mathrm{f}(\mathrm{t})=0$ by $\mathcal{P}_{\mathrm{T}} \mathrm{C}_{0}(\mathbb{R})$. Next, let us introduce the notion of impulsive asymptotically almost periodic functions.

Definition 1.9. Let $\mathrm{T}=\left\{\mathrm{t}_{\mathrm{k}}\right\}_{\mathrm{k} \in \mathbb{Z}} \in \mathcal{T}$. A function $\mathrm{f} \in \mathcal{P}_{\mathrm{T}} \mathrm{C}(\mathbb{R})$ is said to be be asymptotically almost periodic if $\mathrm{f}=\mathrm{g}+\mathrm{h}$, where $\mathrm{g} \in \mathcal{P}_{\mathrm{T}} A \mathrm{P}(\mathbb{R})$ and $\mathrm{h} \in \mathcal{P}_{\mathrm{T}} \mathrm{C}_{0}(\mathbb{R})$. We denote the set of all such functions by $\mathcal{P}_{\mathrm{T}} A A \mathrm{P}(\mathbb{R})$.

Lemma 1.10. Let $\mathrm{T}=\left\{\mathrm{t}_{\mathrm{k}}\right\}_{\mathrm{k} \in \mathbb{Z}} \in \mathcal{T}$. The following assertions hold true:

(i) Let $\mathrm{f}=\mathrm{g}+\mathrm{h} \in \mathcal{P}_{\mathrm{T}} \mathrm{AAP}(\mathbb{R})$, where $\mathrm{g} \in \mathcal{P}_{\mathrm{T}} \mathrm{AP}(\mathbb{R})$ and $\mathrm{h} \in \mathcal{P}_{\mathrm{T}} \mathrm{C}_{0}(\mathbb{R})$. Then

$$
\{g(t): t \in \mathbb{R}\} \subset \overline{\{f(t): t \in \mathbb{R}\}}
$$

(ii) The decomposition of a function $\mathrm{f} \in \mathcal{P}_{\mathrm{T}} \mathrm{AAP}(\mathbb{R})$ is unique.

(iii) $\mathcal{P}_{\mathrm{T}} \mathrm{AAP}(\mathbb{R})$ is a Banach space under the supremum norm.

(iv) Let $\mathrm{f}_{1}, \mathrm{f}_{2} \in \mathcal{P}_{\mathrm{T}} A A P(\mathbb{R})$. Then $\mathrm{f}_{1}+\mathrm{f}_{2} \in \mathcal{P}_{\mathrm{T}} A A \mathrm{P}(\mathbb{R}), \mathrm{f}_{1} \cdot \mathrm{f}_{2} \in \mathcal{P}_{\mathrm{T}} A A \mathrm{P}(\mathbb{R})$ and $\mathrm{f}_{1}(\cdot-\mathrm{a}) \in$ $\mathcal{P}_{\mathrm{T}+\mathrm{a}} A A \mathrm{P}(\mathbb{R})$ for every $\mathrm{a} \in \mathbb{R}$.

(v) Let $\mathrm{f}_{1}, \mathrm{f}_{2} \in \mathcal{P}_{\mathrm{T}} \mathrm{AAP}(\mathbb{R})$. Then $\mathrm{f}_{1} / \mathrm{f}_{2} \in \mathcal{P}_{\mathrm{T}} A A \mathrm{P}(\mathbb{R})$ provided that $\inf _{\mathrm{t} \in \mathbb{R}}\left|\mathrm{f}_{2}(\mathrm{t})\right|>0$.

(vi) Let $\mathrm{f} \in \mathcal{P}_{\mathrm{T}} \mathrm{AAP}(\mathbb{R})$. Then $\mathrm{k} \rightarrow \mathrm{f}\left(\mathrm{t}_{\mathrm{k}}\right)$ belongs to $\mathrm{AAP}(\mathbb{Z})$.

Proof. (i) Since $\mathrm{g}$ is left continuous on $\mathbb{R}$, it suffices to prove that

$$
\{g(t): t \in \mathbb{R}, t \notin T\} \subset \overline{\{f(t): t \in \mathbb{R}\}} .
$$


We prove it by contradiction. Assume that there exists $\mathrm{t}^{\prime} \notin \mathrm{T}$ such that

$$
\varepsilon_{0}:=\inf _{t \in \mathbb{R}}\left|g\left(t^{\prime}\right)-f(t)\right|>0
$$

Let $\delta=\min \left\{\inf _{k \in \mathbb{Z}} \frac{\left|t^{\prime}-t_{k}\right|}{2}, \frac{\varepsilon_{0}}{2}\right\}$. Then, $\delta>0$. It follows from $g \in \mathcal{P}_{\mathrm{T}} A P(\mathbb{R})$ that there exists $l>0$ such that for every $n \in \mathbb{N}$, there is $r_{n} \in\left[n-t^{\prime}, n-t^{\prime}+l\right]$ such that

$$
\left|g\left(t^{\prime}+r_{n}\right)-g\left(t^{\prime}\right)\right|<\delta \leq \frac{\varepsilon_{0}}{2} .
$$

Combing this with $h \in \mathcal{P}_{\mathrm{T}} \mathrm{C}_{0}(\mathbb{R})$, we have

$$
\begin{aligned}
\varepsilon_{0} & \leq\left|g\left(t^{\prime}\right)-f\left(t^{\prime}+r_{n}\right)\right| \\
& \leq\left|g\left(t^{\prime}\right)-g\left(t^{\prime}+r_{n}\right)\right|+\left|h\left(t^{\prime}+r_{n}\right)\right| \\
& \leq \frac{\varepsilon_{0}}{2}+\left|h\left(t^{\prime}+r_{n}\right)\right| \rightarrow \frac{\varepsilon_{0}}{2},
\end{aligned}
$$

which is a contradiction. This completes the proof.

(ii) It suffices to show that 0 has unique decomposition. In fact, letting $0=g+h \in \mathcal{P}_{\top} A A P(\mathbb{R})$, where $g \in \mathcal{P}_{\top} A P(\mathbb{R})$ and $h \in \mathcal{P}_{\top} C_{0}(\mathbb{R})$, it follows from (i) that $g=0$ and thus $h=0$.

(iii) Note that $\mathcal{P}_{\mathrm{T}} \mathrm{C}(\mathbb{R}), \mathcal{P}_{\mathrm{T}} \mathrm{C}_{0}(\mathbb{R})$ and $\mathcal{P}_{\mathrm{T}} \mathrm{AP}(\mathbb{R})$ are all Banach spaces under the supremum norm. Let $\left\{f_{n}\right\} \subset \mathcal{P}_{T} A A P(\mathbb{R})$ be a Cauchy sequence, and $f_{n}=g_{n}+h_{n}$, where $g_{n} \in \mathcal{P}_{T} A P(\mathbb{R})$ and $h_{n} \in \mathcal{P}_{T} C_{0}(\mathbb{R})$. Then, it follows from (i) that $\left\{g_{n}\right\}$ and $\left\{h_{n}\right\}$ are both Cauchy sequences. The remaining proof follows easily.

(iv) The proof follows from (i) and (v) of Lemma 1.7 and the boundedness of every function in $\mathcal{P}_{\mathrm{T}} A \mathrm{P}(\mathbb{R})\left(\right.$ or $\left.\mathcal{P}_{\mathrm{T}} \mathrm{C}_{0}(\mathbb{R})\right)$.

(v) It suffices to prove that $1 / f \in \mathcal{P}_{\top} A A P(\mathbb{R})$ if $f \in \mathcal{P}_{\top} A A P(\mathbb{R})$ with $\inf _{t \in \mathbb{R}}|f(t)|>0$. Let $\mathrm{f}=\mathrm{g}+\mathrm{h} \in \mathcal{P}_{\mathrm{T}} A A P(\mathbb{R})$, where $\mathrm{g} \in \mathcal{P}_{\mathrm{T}} A \mathrm{P}(\mathbb{R})$ and $\mathrm{h} \in \mathcal{P}_{\mathrm{T}} \mathrm{C}_{0}(\mathbb{R})$. Moreover, $\inf _{\mathrm{t} \in \mathbb{R}}|\mathrm{f}(\mathrm{t})|>0$. By (i), there holds $\inf _{\mathbf{t} \in \mathbb{R}}|g(t)|>0$. The remaining proof follows from (ii) of Lemma 1.7 and

$$
\frac{1}{f}=\frac{1}{g}-\frac{h}{f g} .
$$

(vi) The proof follows from (iii) of Lemma 1.7 and $\lim _{k \rightarrow \infty} t_{k}=\infty$.

\section{Linear inhomogenous equation}

Throughout the rest of this paper, if there is no special statement, we assume that $T=\left\{t_{k}\right\}_{k \in \mathbb{Z}} \in \mathcal{T}$ and the set of sequences $\left\{T_{j}\right\}_{j \in \mathbb{Z}}$ is equi-almost periodic, where $T_{j}=\left\{t_{k}^{j}: t_{k}^{j}=t_{k+j}-t_{k}, k \in \mathbb{Z}\right\}$ for every $j \in \mathbb{Z}$. By [9, Lemma 22], the following limit exists:

$$
\lim _{t-s \rightarrow+\infty} \frac{i(s, t)}{t-s}
$$


where $i(s, t)$ is the number of the terms of $T \cap[s, t]$. We denote

$$
p=\lim _{t \rightarrow s \rightarrow+\infty} \frac{i(s, t)}{t-s}
$$

Now, let us first consider the following linear inhomogenous equation:

$$
\begin{cases}x^{\prime}(t)=-a(t) x(t)+f(t), & t \neq t_{k} \\ \left.\Delta x\right|_{t=t_{k}}=c_{k} x\left(t_{k}\right)+I_{k}, & k \in \mathbb{Z}\end{cases}
$$

where $a \in A P(\mathbb{R}), c_{k}$ is an almost periodic sequence, and $I_{k}$ is an asymptotically almost periodic sequence. Moreover, let $T^{\prime} \in \mathcal{T}$ with $T \subset T^{\prime}$ and $f \in \mathcal{P}_{T}, A A P(\mathbb{R})$. Denote

$$
M(a)=\lim _{T \rightarrow+\infty} \frac{1}{T} \int_{0}^{T} a(t) d t, \quad \beta=\sup _{k \in \mathbb{Z}}\left|1+c_{k}\right|,
$$

and

$$
X(t, s)=\left\{\begin{array}{l}
\exp \left(-\int_{s}^{t} a(u) d u\right), \quad t_{k-1}<s \leq t \leq t_{k}, \\
\prod_{i=m}^{k}\left(1+c_{i}\right) \cdot \exp \left(-\int_{s}^{t} a(u) d u\right), \quad t_{m-1}<s \leq t_{m} \leq t_{k}<t \leq t_{k+1} .
\end{array}\right.
$$

Definition 2.1. Let $\mathrm{T}=\left\{\mathrm{t}_{\mathrm{k}}\right\}_{\mathrm{k} \in \mathbb{Z}} \in \mathcal{T}, \mathrm{T}^{\prime} \in \mathcal{T}$ with $\mathrm{T} \subset \mathrm{T}^{\prime}$ and $\mathrm{f} \in \mathcal{P}_{\mathrm{T}}, \mathrm{C}(\mathbb{R})$. We call that $\mathrm{x}$ is a global solution of equation (2.1) if

$$
\begin{cases}x^{\prime}(t)=-a(t) x(t)+f(t), & t \notin T^{\prime} \\ \left.\Delta x\right|_{t=t_{k}}=c_{k} x\left(t_{k}\right)+I_{k}, & k \in \mathbb{Z}\end{cases}
$$

We have the following results about equation (2.1):

Theorem 2.2. Let $\mathrm{M}(\mathrm{a})>\mathrm{p} \cdot \ln \beta$. Then, equation (2.1) has a unique global solution $\overline{\mathrm{x}}$ in $\mathcal{P}_{\mathrm{T}} A A \mathrm{P}(\mathbb{R})$. Moreover, we have

$$
\bar{x}(t)=\int_{-\infty}^{t} X(t, s) f(s) d s+\sum_{t_{k}<t} X\left(t, t_{k}\right) I_{k}, \quad t \in \mathbb{R} .
$$

Proof. We give the proof by four steps.

Step 1. There exist $M, \omega>0$ such that

$$
|X(t, s)| \leq M e^{-\omega(t-s)}
$$

for all $t, s \in \mathbb{R}$ with $t \geq s$. 
It suffices to prove the above inequality for $t-s$ being sufficiently large. Let $\alpha \in(0, M(a))$ and $q>p$ be such that $\omega:=\alpha-q \ln \beta>0$. It follows that

$$
\alpha(t-s) \leq \int_{s}^{t} a(u) d u, \quad i(s, t) \leq q(t-s),
$$

for all $t, s \in \mathbb{R}$ with $t-s$ being sufficiently large. Then, we have

$$
|X(t, s)|=\left\{\begin{array}{l}
\left|e^{-\int_{s}^{t} a(u) d u}\right| \leq e^{-\alpha(t-s)}, \quad t_{k-1}<s \leq t \leq t_{k} \\
\left|\prod_{i=m}^{k}\left(1+c_{i}\right) \cdot e^{-\int_{s}^{t} a(u) d u}\right| \leq \beta^{q(t-s)} \cdot e^{-\alpha(t-s)}=e^{-\omega(t-s)}, \quad t_{m-1}<s \leq t_{m} \leq t_{k}<t \leq t_{k+1} .
\end{array}\right.
$$

Step 2. $\bar{x}$ is a global solution of equation (2.1).

By Step 1 and direct calculations, one can obtain

$$
\bar{x}^{\prime}(t)=-a(t) \bar{x}(t)+f(t), \quad t \notin T^{\prime} .
$$

Moreover, it is not difficult to verify that $\left.\Delta \bar{x}\right|_{t=t_{k}}=c_{k} \bar{x}\left(t_{k}\right)+I_{k}$ for $k \in \mathbb{Z}$. In addition, for every $\mathrm{t} \in \mathrm{T}^{\prime} \backslash \mathrm{T}$, there holds

$$
\bar{x}_{+}^{\prime}(t)=-a(t) \bar{x}(t)+f(t+0), \quad \bar{x}_{-}^{\prime}(t)=-a(t) \bar{x}(t)+f(t) .
$$

Step 3. $\bar{x} \in \mathcal{P}_{\mathrm{T}} A A P(\mathbb{R})$.

Let $f=g+h$, where $g \in \mathcal{P}_{\mathrm{T}}, A P(\mathbb{R})$ and $h \in \mathcal{P}_{\mathrm{T}}, \mathrm{C}_{0}(\mathbb{R})$. Moreover, let $\mathrm{I}_{\mathrm{k}}=\mathrm{J}_{\mathrm{k}}+\mathrm{L}_{\mathrm{k}}$, where $k \rightarrow J_{k}$ belongs to $\operatorname{AP}(\mathbb{Z})$ and $k \rightarrow L_{k}$ belongs to $C_{0}(\mathbb{Z})$. Then, we have

$$
\begin{aligned}
\bar{x}(t) & =\int_{-\infty}^{t} X(t, s) f(s) d s+\sum_{t_{k}<t} X\left(t, t_{k}\right) I_{k} \\
& =\int_{-\infty}^{t} X(t, s) g(s) d s+\int_{-\infty}^{t} X(t, s) h(s) d s+\sum_{t_{k}<t} X\left(t, t_{k}\right) J_{k}+\sum_{t_{k}<t} X\left(t, t_{k}\right) L_{k} \\
& :=\overline{\chi_{1}}(t)+\overline{\chi_{2}}(t)+\overline{\chi_{3}}(t)+\overline{\chi_{4}}(t) .
\end{aligned}
$$

It follows from $\left[9\right.$, Theorem 81] that $\overline{x_{1}} \in \mathcal{P}_{\mathrm{T}}, A P(\mathbb{R})$ and $\overline{x_{3}} \in \mathcal{P}_{\mathrm{T}} A P(\mathbb{R})$. Noting that $\overline{x_{1}}$ is continuous, we conclude that $\overline{x_{1}} \in \operatorname{AP}(\mathbb{R})$. Noting that

$$
\overline{x_{2}}(t)=\int_{0}^{+\infty} X(t, t-s) h(t-s) d s,
$$

by using Step 1 and $h \in \mathcal{P}_{\mathrm{T}}, \mathrm{C}_{0}(\mathbb{R})$, one can conclude that $\overline{\mathrm{x}_{2}}$ is continuous and $\lim _{\mathrm{t} \rightarrow \infty} \overline{\mathrm{x}_{2}}(\mathrm{t})=0$. Again by using Step 1 and $k \rightarrow L_{k}$ belonging to $C_{0}(\mathbb{Z})$, one can conclude that $\overline{\chi_{4}} \in \mathcal{P}_{\mathrm{T}} C_{0}(\mathbb{R})$. Combining all the above proof, we have $\bar{x} \in \mathcal{P}_{\top} A A P(\mathbb{R})$.

Step 4. Uniqueness. 
Let $y \in \mathcal{P}_{\mathrm{T}} A A P(\mathbb{R})$ satisfying (2.2). Noting that $y$ is continuous on $\mathrm{T}^{\prime} \backslash \mathrm{T}$, by using equation (2.1), it is not difficult to verify that

$$
y(t)=X(t, s) y(s)+\int_{s}^{t} X(t, s) f(s) d s+\sum_{s \leq t_{i}<t} X\left(t, t_{i}\right) I_{i},
$$

for all $t, s \in \mathbb{R}$ with $t \geq s$. Letting $s \rightarrow-\infty$, noting that $y$ is bounded and $|X(t, s)| \leq M e^{-\omega(t-s)}$, we have

$$
y(t)=\int_{-\infty}^{t} X(t, s) f(s) d s+\sum_{t_{k}<t} X\left(t, t_{k}\right) I_{k}=\bar{x}(t), \quad t \in \mathbb{R} .
$$

This completes the proof.

\section{$3 \quad$ Existence and stability}

Now, let us discuss the existence and stability of asymptotically almost periodic solution for equation (1.2). For convenience, we only consider the case of $n>1$. We first list some assumptions.

(H1) $a \in A P(\mathbb{R})$, and $c_{k}$ is an almost periodic sequence with $c_{k} \geq-1$ for all $k \in \mathbb{Z}$.

$(\mathrm{H} 2) \mathrm{b} \in \mathcal{P}_{\mathrm{T}} A A P(\mathbb{R})$ is nonnegative, and for every $\mathrm{x} \in \mathbb{R}^{+}, \mathrm{k} \rightarrow \mathrm{I}_{\mathrm{k}}(\mathrm{x})$ is a nonnegative asymptotically almost periodic sequence. Moreover, there exists a constant $\mathrm{L}>0$ such that for all $x, y \in \mathbb{R}^{+}$and $k \in \mathbb{Z}$, there holds $\left|I_{k}(x)-I_{k}(y)\right| \leq L|x-y|$.

(H3) There exist $M, \omega>0$ such that $|X(t, s)| \leq M e^{-\omega(t-s)}$ for all $t, s \in \mathbb{R}$ with $t \geq s$.

(H4) $\frac{M\|b\|}{\omega} \cdot \frac{n^{2}-1}{4 n} \sqrt[n]{\frac{n+1}{n-1}}+\frac{M L}{1-e^{-\omega \theta}}<1$, where $\theta=\inf _{k \in \mathbb{Z}}\left|t_{k+1}-t_{k}\right|$.

Remark 3.1. It follows from Step 1 of the proof for Theorem 2.2 that $(H 3)$ holds if $M(a)>p \cdot \ln \beta$.

Before presenting our results, we need to clarify that our definition of solution for equation (1.2) has a slight difference with the classical definition of solution for equation (1.2).

Definition 3.2. We call that a function $x \in \mathcal{P}_{\mathrm{T}} \mathrm{C}(\mathbb{R})$ is a global solution of equation (1.2) if

$$
\begin{cases}x^{\prime}(t)=-a(t) x(t)+\frac{b(t)}{1+x^{n}(t-\tau)}, & t \neq t_{k}, \quad t \neq t_{k}+\tau, \\ \left.\Delta x\right|_{t=t_{k}}=c_{k} x\left(t_{k}\right)+I_{k}\left(x\left(t_{k}\right)\right), \quad & k \in \mathbb{Z} .\end{cases}
$$

Moreover, we need to recall a Gronwall inequality to discuss the stability.

Lemma 3.3. [9, Lemma 2] Let $\mathfrak{u} \in \mathcal{P}_{\mathrm{T}} \mathrm{C}(\mathbb{R})$ be nonnegative, $\mathrm{s} \in \mathbb{R}$ and for all $\mathrm{t} \geq \mathrm{s}$,

$$
u(t) \leq C+\int_{s}^{t} \gamma u(x) d x+\sum_{s \leq t_{k}<t} \beta u\left(t_{k}\right)
$$


where $\mathrm{C} \geq 0, \beta \geq 0$ and $\gamma>0$ are all constants. Then, there exists a constant $\mathrm{C}^{\prime}>0$ such that for all $\mathrm{t} \geq \mathrm{s}$,

$$
u(t) \leq C^{\prime}(1+\beta)^{i(s, t)} e^{\gamma(t-s)} .
$$

Theorem 3.4. Assume that (H1)-(H4) hold. Then, equation (1.2) has a unique nonnegative asymptotically almost periodic solution. Moreover, the asymptotically almost periodic solution of equation (1.2) is exponentially stability provided that

$$
p \ln (1+L M)+\frac{n^{2}-1}{4 n} \sqrt[n]{\frac{n+1}{n-1}} M e^{\omega \tau}\|b\|<\omega .
$$

Proof. Let $\varphi \in \mathcal{P}_{\mathrm{T}} A A \mathrm{P}(\mathbb{R})$ with $\inf _{\mathrm{t} \in \mathbb{R}} \varphi(\mathrm{t}) \geq 0$. Consider

$$
\begin{cases}x^{\prime}(t)=-a(t) x(t)+\frac{b(t)}{1+\varphi^{n}(t-\tau)}, & t \neq t_{k}, \\ \left.\Delta x\right|_{t=t_{k}}=c_{k} x\left(t_{k}\right)+I_{k}\left(\varphi\left(t_{k}\right)\right), & k \in \mathbb{Z} .\end{cases}
$$

By Lemma 1.10, we have

$$
\frac{\mathrm{b}(\cdot)}{1+\varphi^{\mathfrak{n}}(\cdot-\tau)} \in \mathcal{P}_{\mathrm{T} \cup(\mathrm{T}+\tau)} \operatorname{AAP}(\mathbb{R})
$$

Again by Lemma 1.10, we get $k \rightarrow \varphi\left(t_{k}\right)$ belongs to $\operatorname{AAP}(\mathbb{Z})$. Then, by (H2), it is not difficult to show that $\mathrm{k} \rightarrow \mathrm{I}_{\mathrm{k}}\left(\varphi\left(\mathrm{t}_{\mathrm{k}}\right)\right)$ belongs to $\operatorname{AAP}(\mathbb{Z})$.

Now, by Theorem 2.2, we know that for every $\varphi \in \mathcal{P}_{\top} A A P(\mathbb{R})$ with $\inf _{t \in \mathbb{R}} \varphi(t) \geq 0$, equation (3.2) has a unique global solution $x^{\varphi} \in \mathcal{P}_{\mathrm{T}} A A P(\mathbb{R})$, which satisfies

$$
x^{\varphi}(t)=\int_{-\infty}^{t} X(t, s) \frac{b(s)}{1+\varphi^{\mathfrak{n}}(s-\tau)} d s+\sum_{t_{k}<t} X\left(t, t_{k}\right) I_{k}\left(\varphi\left(t_{k}\right)\right), \quad t \in \mathbb{R} .
$$

Define a mapping $\mathcal{F}$ on $\Omega=\left\{\varphi \in \mathcal{P}_{\mathrm{T}} A A P(\mathbb{R}): \inf _{\mathrm{t} \in \mathbb{R}} \varphi(\mathrm{t}) \geq 0\right\}$ by

$$
\mathcal{F}(\varphi)(t)=\int_{-\infty}^{t} X(t, s) \frac{b(s)}{1+\varphi^{n}(s-\tau)} d s+\sum_{t_{k}<t} X\left(t, t_{k}\right) I_{k}\left(\varphi\left(t_{k}\right)\right), \quad t \in \mathbb{R}, \varphi \in \Omega .
$$

It is easy to see that $\Omega$ is a closed subset in $\mathcal{P}_{\mathrm{T}} A A P(\mathbb{R})$ and $\mathcal{F}(\Omega) \subset \Omega$ by the assumptions.

Noting that

$$
\sup _{u \geq 0}\left|\left(\frac{1}{1+u^{n}}\right)^{\prime}\right|=\sup _{u \geq 0} \frac{n u^{n-1}}{\left(1+u^{n}\right)^{2}} \leq \frac{n^{2}-1}{4 n} \sqrt[n]{\frac{n+1}{n-1}},
$$

we conclude by mean value theorem that

$$
\left|\frac{1}{1+x^{n}}-\frac{1}{1+y^{n}}\right| \leq \frac{n^{2}-1}{4 n} \sqrt[n]{\frac{n+1}{n-1}} \cdot|x-y|, \quad x, y \geq 0
$$

Combining this with

$$
|X(t, s)| \leq M e^{-\omega(t-s)}, \quad t, s \in \mathbb{R}, t \geq s,
$$


for all $\varphi, \psi \in \Omega$, there holds

$$
\begin{aligned}
& \|\mathcal{F}(\varphi)-\mathcal{F}(\psi)\| \\
= & \sup _{\mathrm{t} \in \mathbb{R}}|\mathcal{F}(\varphi)(\mathrm{t})-\mathcal{F}(\psi)(\mathrm{t})| \\
\leq & \sup _{\mathrm{t} \in \mathbb{R}} \int_{-\infty}^{\mathrm{t}} M e^{-\omega(\mathrm{t}-s)} \mathrm{b}(\mathrm{s}) \mathrm{ds} \cdot \frac{\mathrm{n}^{2}-1}{4 n} \sqrt[n]{\frac{n+1}{n-1}}\|\varphi-\psi\|+\sup _{\mathrm{t} \in \mathbb{R}} \sum_{\mathbf{t}_{\mathrm{k}}<\mathrm{t}} M e^{-\omega\left(\mathrm{t}-\mathrm{t}_{\mathrm{k}}\right)} \cdot \mathrm{L}\|\varphi-\psi\| \\
\leq & \frac{\mathrm{M}\|\mathrm{b}\|}{\omega} \cdot \frac{\mathrm{n}^{2}-1}{4 n} \sqrt[\mathfrak{n}]{\frac{\mathrm{n}+1}{n-1}}\|\varphi-\psi\|+\sum_{\mathrm{k}=0}^{\infty} M e^{-k \omega \theta} \cdot \mathrm{L}\|\varphi-\psi\| \\
= & \left(\frac{M\|\mathrm{~b}\|}{\omega} \cdot \frac{\mathrm{n}^{2}-1}{4 n} \sqrt[n]{\frac{n+1}{n-1}}+\frac{M L}{1-e^{-\omega \theta}}\right) \cdot\|\varphi-\psi\| .
\end{aligned}
$$

Then, by (H4), $\mathcal{F}$ is a contraction and thus $\mathcal{F}$ has a unique fixed point in $\Omega$, i.e., equation (1.2) has a unique nonnegative asymptotically almost periodic solution.

Let $x$ be the above asymptotically almost periodic solution of equation (1.2). Next, let us discuss the stability of $x$. Let $y$ be an arbitrary solution of equation $(1.2)$ on $\left[t_{0},+\infty\right)$. It is not difficult to verify that

$$
x(t)=X\left(t, t_{0}\right) x\left(t_{0}\right)+\int_{t_{0}}^{t} X(t, s) \frac{b(s)}{1+x^{n}(s-\tau)} d s+\sum_{t_{0} \leq t_{k}<t} X\left(t, t_{k}\right) I_{k}\left(x\left(t_{k}\right)\right), \quad t \geq t_{0},
$$

and

$$
y(t)=X\left(t, t_{0}\right) y\left(t_{0}\right)+\int_{t_{0}}^{t} X(t, s) \frac{b(s)}{1+y^{n}(s-\tau)} d s+\sum_{t_{0} \leq t_{k}<t} X\left(t, t_{k}\right) I_{k}\left(y\left(t_{k}\right)\right), \quad t \geq t_{0} .
$$

Letting $u(t)=x(t)-y(t)$, we have for all $t \geq t_{0}$,

$|u(t)| \leq M e^{-\omega\left(t-t_{0}\right)}\left|u\left(t_{0}\right)\right|+N\|b\| \int_{t_{0}}^{t} M e^{-\omega(t-s)}|x(s-\tau)-y(s-\tau)| d s+L \sum_{t_{0} \leq t_{k}<t} M e^{-\omega\left(t-t_{k}\right)}\left|u\left(t_{k}\right)\right|$,

where $N=\frac{n^{2}-1}{4 n} \sqrt[n]{\frac{n+1}{n-1}}$. Letting $v(t)=e^{\omega t}|u(t)|$, we get for all $t \geq t_{0}$,

$$
\begin{aligned}
v(t) & \leq M v\left(t_{0}\right)+N M\|b\| \int_{t_{0}}^{t} e^{\omega s}|x(s-\tau)-y(s-\tau)| d s+L M \sum_{t_{0} \leq t_{k}<t} v\left(t_{k}\right) \\
& =M v\left(t_{0}\right)+N M\|b\| \int_{t_{0}-\tau}^{t-\tau} e^{\omega \tau} e^{\omega s}|x(s)-y(s)| d s+L M \sum_{t_{0} \leq t_{k}<t} v\left(t_{k}\right) \\
& \leq M v\left(t_{0}\right)+N M e^{\omega \tau}\|b\| \int_{t_{0}-\tau}^{t} v(s) d s+L M \sum_{t_{0} \leq t_{k}<t} v\left(t_{k}\right) \\
& \leq C+N M e^{\omega \tau}\|b\| \int_{t_{0}}^{t} v(s) d s+L M \sum_{t_{0} \leq t_{k}<t} v\left(t_{k}\right),
\end{aligned}
$$


where $C=M v\left(t_{0}\right)+N M e^{\omega \tau} \tau \cdot \max _{s \in\left[t_{0}-\tau, t_{0}\right]} v(s)$. Combining this with Lemma 3.3, we have

$$
v(t) \leq C^{\prime}(1+L M)^{i\left(t_{0}, t\right)} \cdot e^{N M e^{\omega \tau}\|b\|\left(t-t_{0}\right)}, \quad t \geq t_{0} .
$$

By (3.1), we can choose $q>p$ such that

$$
\mathrm{q} \ln (1+\mathrm{LM})+\mathrm{NM} \mathrm{e}^{\omega \tau}\|\mathrm{b}\|<\omega .
$$

Noting the definition of $p$, we deduce that for sufficiently large $t$, there holds

$$
(1+\mathrm{LM})^{i\left(\mathrm{t}_{0}, \mathrm{t}\right)} \leq(1+\mathrm{LM})^{\mathrm{q}\left(\mathrm{t}-\mathrm{t}_{0}\right)}=e^{\mathrm{q}\left(\mathrm{t}-\mathrm{t}_{0}\right) \ln (1+\mathrm{LM})},
$$

which means that

$$
v(t) \leq C^{\prime} e^{\left[q \ln (1+L M)+N M e^{\omega \tau}\|b\|\right]\left(t-t_{0}\right)},
$$

i.e.,

$$
|x(t)-y(t)| \leq C^{\prime} e^{-\left[\omega-q \ln (1+L M)+N M e^{\omega \tau}\|b\|\right]\left(t-t_{0}\right)} .
$$

Thus, $y(t)$ converges exponentially to $x(t)$ when $t \rightarrow+\infty$.

At last, we give a simple example to illustrate our results, which does not aim at generality.

Example 3.5. Let $n=2$,

$$
\begin{gathered}
t_{k}=k+\frac{1}{4}|\sin k-\sin \sqrt{2} k|, \quad a(t)=20+20(\sin 600 t+\sin 600 \pi t), \\
b(t)=\frac{1}{10}\left(|\cos t+\cos \pi t|+\frac{1}{1+t^{2}}\right), \quad c_{k}=1-\frac{\sin k+\sin \sqrt{3} k+e^{-k^{2}}}{3},
\end{gathered}
$$

and

$$
I_{k}(x)=\frac{\cos ^{2} k+\cos ^{2} \sqrt{3} k+e^{-k^{2}}}{10}|\cos x| .
$$

It is easy to verify that $(\mathrm{H} 1)$ and $(\mathrm{H} 2)$ hold with $\mathrm{L}=\frac{3}{10}$. Also, one can show that (H3) holds with $M=\sqrt[10]{e}$ and $\omega=18$. Moreover, we have $\|\mathrm{b}\|=\frac{3}{10}$ and

$$
\theta=\inf _{k \in \mathbb{Z}}\left|t_{k+1}-t_{k}\right| \in\left[\frac{1}{2}, 1\right], \quad p=\lim _{t-s \rightarrow+\infty} \frac{i(s, t)}{t-s} \leq 2 .
$$

By direct calculations, one can get

$$
\frac{M\|b\|}{\omega} \cdot \frac{n^{2}-1}{4 n} \sqrt[n]{\frac{n+1}{n-1}}+\frac{M L}{1-e^{-\omega \theta}}<1
$$

i.e., (H4) holds. Also, one can verify that

$$
p \ln (1+L M)+\frac{n^{2}-1}{4 n} \sqrt[n]{\frac{n+1}{n-1}} M\|b\|<\omega
$$

which means that (3.1) holds if $\tau$ is sufficiently small.

Remark 3.6. In the above example, one can easily see that $\inf _{t \in \mathbb{R}} a(t)<0$ and $c_{k}>0$ for all $k \in \mathbb{Z}$. 


\section{Acknowledgements}

H.-S. Ding acknowledges support from NSFC (11461034), the Program for Cultivating Young Scientist of Jiangxi Province (20133BCB23009), and the NSF of Jiangxi Province (20143ACB21001).

Received: December 2014. Accepted: January 2015.

\section{References}

[1] J. O. Alzabut, J. J. Nieto, G. Tr. Stamov, Existence and exponential stability of positive almost periodic solutions for a model of hematopoiesis, Bound. Value Probl. 2009, Art. ID 127510, 10 pp.

[2] C. Corduneanu, Almost Periodic Functions, 2nd edition, Chelsea, New york, 1989.

[3] T. Diagana, Pseudo Almost Periodic Functions in Banach Spaces, Nova Science, New York, 2007.

[4] H. S. Ding, G. M. N'Guérékata, J. J. Nieto, Weighted pseudo almost periodic solutions for a class of discrete hematopoiesis model, Rev. Mat. Complut. 26 (2013), 427-443.

[5] M. Fréchet, Fonctions asymptotiquement presque périodiques, Revue Scientifique (Revue Rose Illustrée) 79 (1941), 341-354.

[6] H. R. Henríquez, B. D. Andrade, M. Rabelo, Existence of almost periodic solutions for a class of abstract impulsive differential equations, ISRN Mathematical Analysis, Volume 2011, Article ID 632687, 21 pages.

[7] B. Liu, New results on the positive almost periodic solutions for a model of hematopoiesis, Nonlinear Anal. Real World Appl. 17 (2014), 252-264.

[8] M. C. Mackey, L. Glass, Oscillation and chaos in physiological control system, Science 197 (1977), 287-289.

[9] A. M. Samoilenko, N. A. Perestyuk, Impulsive Differential Equations, World Scientific, Singapore, 1995.

[10] S. H. Saker, J. O. Alzabut, On the impulsive delay hematopoiesis model with periodic coefficients, Rocky Mountain J. Math. 39 (2009), 1657-1688.

[11] G. T. Stamov, Almost Periodic Solutions of Impulsive Differential Equations, Springer-Verlag, Berlin, 2012.

[12] Z. Yao, Almost periodicity of impulsive hematopoiesis model with infinite delay, J. Nonlinear Sci. Appl. 8 (2015), 856-865. 